\title{
Percutaneous trigeminal tractotomy-nucleotomy with use of intraoperative computed tomography and general anesthesia: report of 2 cases
}

\author{
Eric M. Thompson, M.D., Kim J. Burchiel, M.D., and Ahmed M. Raslan, M.D. \\ Department of Neurological Surgery, Oregon Health \& Science University, Portland, Oregon
}

\begin{abstract}
For confirming the correct location of the radiofrequency electrode before creation of a lesion, percutaneous CTguided trigeminal tractotomy-nucleotomy is most commonly performed with the patient prone and awake. However, for patients whose facial pain and hypersensitivity are so severe that the patients are unable to rest their face on a support (as required with prone positioning), awake CT-guided tractotomy-nucleotomy might not be feasible. The authors describe 2 such patients, for whom percutaneous intraoperative CT-guided tractotomy-nucleotomy under general anesthesia was successful. One patient was a 79-year-old man with profound left facial postherpetic neuralgia, who was unable to tolerate awake CT-guided tractotomy-nucleotomy, and the other was a 45-year-old woman with intractable hemicranial pain that developed after a right frontal lesionectomy for epilepsy. Each patient underwent a percutaneous intraoperative CT-guided tractotomy-nucleotomy under general anesthesia. No complications occurred, and each patient reported excellent pain relief for up to 6 and 3 months after surgery, respectively. Percutaneous intraoperative CT-guided tractotomy-nucleotomy performed on anesthetized patients is effective for facial postherpetic neuralgia and postoperative hemicranial neuralgia.

(http://thejns.org/doi/abs/10.3171/2013.6.FOCUS13218)
\end{abstract}

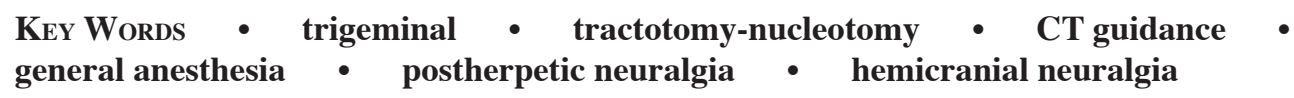

\begin{abstract}
A LTHOUGH not commonly used, tractotomy-nucleotomy is a viable treatment option for patients with malignancy-related facial pain, neuropathic facial pain, postherpetic neuralgia, glossopharyngeal neuralgia, geniculate neuralgia, and refractory trigeminal neuralgia. ${ }^{3,5}$ Postherpetic neuralgia is an especially severe and difficult pain syndrome to treat and is occasionally associated with facial allodynia and hyperalgesia. Tractotomy has a durable success rate of greater than $80 \% .3,5$ The procedure is typically performed with CT guidance in an awake and prone patient. Primary complications include temporary ataxia and motor dysfunction., ${ }^{3,5}$

Some patients, however, have facial pain so profound that they are unable to tolerate awake prone positioning; for these patients, CT-guided tractotomy-nucleotomy might not be feasible, and some neurosurgeons might opt for the more invasive nucleus caudalis dorsal root entry zone procedure. We present 2 cases: one of a postherpetic neuralgia patient for whom awake CT-guided tractotomynucleotomy failed because of intolerance to pain and another of a patient with postoperative hemicranial neuralgia. Each patient underwent successful intraoperative CT-guided tractotomy-nucleotomy while under general anesthesia.
\end{abstract}

\section{Cases Reports}

Case 1

A 79-year-old man sought care after a 7-year history of profound left-sided facial pain, particularly at the ophthalmic division of the trigeminal nerve, resulting from facial herpes zoster (shingles). He reported dull, aching background pain and episodic sharp, burning pain that was exacerbated by light touch. Previous treatments consisted of oral pregabalin and left supraorbital nerve stimulation, both of which minimally relieved the pain. Neurological examination results were nonfocal.

For this patient with a diagnosis of medically refractory postherpetic neuralgia, a decision was made to perform a left-sided CT-guided tractotomy-nucleotomy with the patient awake. However, during the procedure, the patient was unable to tolerate the facial pain caused by the CT scanner headrest; despite aggressive premedication and numerous attempts to reposition and pad the patient's face, the procedure was aborted. A decision was then made to proceed with an intraoperative CT-guided tractotomy-nucleotomy with the patient under general anesthesia and use of somatosensory and motor evoked potentials of the extremities and of the lower cranial nerves 
(cranial nerves X-XII). Approximately 30 minutes before induction of general anesthesia, a lumbar puncture was performed and $12 \mathrm{ml}$ of 300 iohexol (Omnipaque, GE Health Care) was injected.

General anesthesia was induced, and an NIM EMG endotracheal tube (Medtronic) was placed for monitoring of the vagus nerve. For monitoring of somatosensory and motor evoked potentials, electrodes were placed in all 4 extremities, the tongue, and the trapezius muscle. The patient was placed prone and the neck was fixed in flexion in a DORO (Pro Med Instruments) radiolucent skull clamp. The clamp was placed low on the skull and configured so that the intraoperative CT scanner could scan down to the upper cervical spine (Fig. 1A). Under the guidance of a CereTom CT scanner (NeuroLogica Corp.), a percutaneous needle was inserted into the skin approximately $2 \mathrm{~cm}$ left of the midline between the occiput and C-1. A thin, 1.25 -mm-wide field-of-view (FOV) scan through the foramen magnum to the bottom of $\mathrm{C}-1$ was acquired (Fig. 1B). The skin-to-dura distance and the diameters of the upper cervical spinal cord were determined. The skin-todura distance was marked on the introducer cannula for the Levin-Cosman cordotomy electrode system ${ }^{4}$ (Cosman Medical). The electrode depth was adjusted to project 3 $\mathrm{mm}$ from the introducer metal cannula. The laser on the CT scanner was used to mark this point on the patient's skin, and the skin was sterilely prepared and draped. Serial CT scans were used to guide the tip of the Levin-Cosman cordotomy electrode radiofrequency needle to the dorsal surface of the spinal cord, two-thirds of the distance from the midline to the equator of the spinal cord (Fig. 1C), which represents the surface anatomy of the trigeminal tract and the underlying trigeminal nucleus as described by Kanpolat et al. ${ }^{2}$ Impedance was measured by using a Radionics RFG-3C generator (formerly Radionics Inc.) (Fig. 1D) and was 300-400 $\Omega$ in the subarachnoid space. As the spinal cord was entered, impedance increased to $1.4 \mathrm{~K} \Omega$. Another CT scan was used to confirm correct electrode positioning. The tip of the Levin-Cosman cordotomy electrode was stimulated at approximately $0.1 \mathrm{~V}$, which resulted in increased heart rate, suggestive of significant pain. Neuromonitoring was used during the procedure; however, the CT scanner and the Radionics generator produced enough electrical artifact to render neuromonitoring results unusable. Two lesions were then created, one at $90^{\circ} \mathrm{C}$ for 90 seconds and one at $95^{\circ} \mathrm{C}$ for 90 seconds. The patient was awakened from general anesthesia and extubated without problem. The patient immediately noted a slight decrease in touch-induced pain on the left side of his face, and the next day, he reported complete relief of his facial pain. Notably, he did experience mild ataxia and numbness of the left arm, which significantly improved 2 days after discharge. Six months after the procedure, the patient rated his facial pain as 2/10; before the procedure, he had rated it at 9/10.

Case 2

A 45-year-old woman sought care for right hemicranial pain in both trigeminal and occipital nerve distributions, which had developed after surgical resection of a right frontal seizure focus 13 years earlier. The patient had previously undergone occipital nerve stimulation and
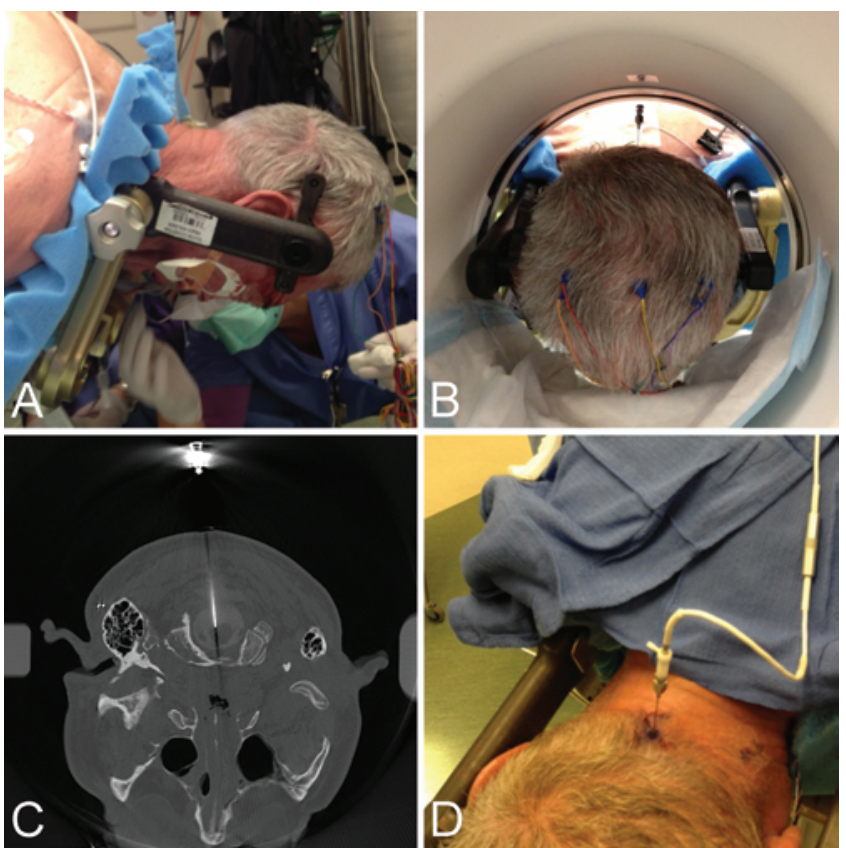

Fig. 1. Case 1. Steps of a percutaneous trigeminal tractotomy-nucleotomy performed using intraoperative CT and general anesthesia. A: The patient is placed in rigid 3-point fixation in the prone position with the head flexed. The skull clamp is placed as inferiorly as possible to allow the plane of the CT scanner to reach C-2. B: The percutaneous needle is inserted $2 \mathrm{~cm}$ lateral to the midline between $\mathrm{C}-1$ and the foramen magnum. Under CT guidance, it is directed to the trigeminal tract of the trigeminal nucleus, two-thirds of the distance from the midline to the equator of the spinal cord. $\quad$ C: The final position of the electrode tip is approximately $3 \mathrm{~mm}$ anterior to the dorsal surface of the cord and $6 \mathrm{~mm}$ lateral from the midline. D: Impedance is then assessed to ensure proper positioning of the electrode within the spinal cord, and 2 lesions are made.

right C-2 ganglionectomy 9 and 7 years earlier, respectively. The patient reported considerable pain that was resistant to a drug regimen consisting of carbamazepine, gabapentin, escitalopram oxalate, acetaminophen/hydrocodone, and diclofenac. Neurological examination findings were nonfocal. Neuromonitoring was not used for this patient because of the significant electrical artifact problems encountered in Case 1. Initially, the patient was placed prone on the DORO radiolucent skull clamp; however, because of her physique, we were unable to visualize to C-1. Therefore, the patient was placed prone on a NeuroLogica Scan Board (NeuroLogica Corp.) (Fig. 2) with her shoulders gently retracted inferiorly with tape, which enabled visualization to C-2. As was done in Case 1, a CereTom CT scanner was used to plan the skin insertion site of the percutaneous needle approximately $2 \mathrm{~cm}$ left of the midline between the occiput and C-1. A thin, 1.25-mm-wide FOV scan through the foramen magnum to the bottom of C-1 was obtained. The skin-to-dura distance and the diameters of the upper cervical spinal cord were determined. The skin-to-dura distance was marked on the introducer cannula for the Levin-Cosman cordotomy electrode system. ${ }^{4}$ The electrode depth was adjusted to project $3 \mathrm{~mm}$ from the introducer metal cannula. The laser on the CT scanner was used to mark this point on 


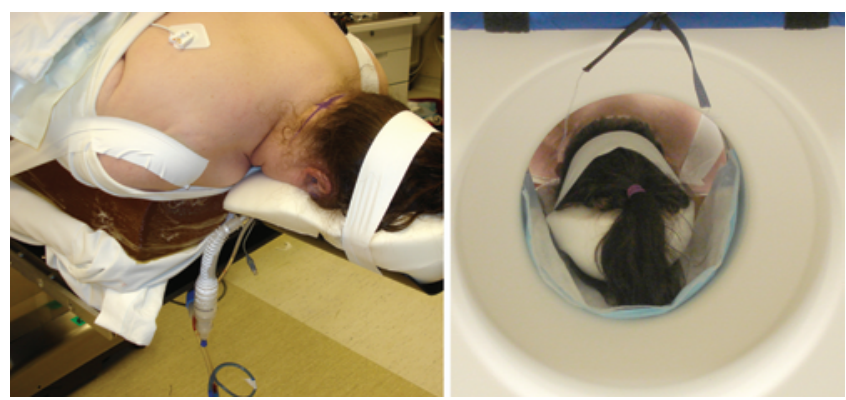

Fig. 2. Case 2. Positioning without using rigid 3-point fixation. Left: The patient is placed on chest rolls to enable head flexion on the NeuroLogica Scan Board. Right: The head is secured with tape, and the shoulders are gently held inferiorly to facilitate entry into the CereTom CT scanner and visualization down to $\mathrm{C}-2$.

the patient's skin, and the skin was sterilely prepared and draped. Serial CT scans were used to guide the tip of the Levin-Cosman cordotomy electrode radiofrequency needle to the dorsal surface of the spinal cord, two-thirds of the distance from the midline to the equator of the spinal cord. Impedance was measured by using a Radionics RFG3C generator and was approximately $300 \Omega$ in the subarachnoid space. As the spinal cord was entered, impedance increased to $1.2 \mathrm{~K} \Omega$. Another CT scan was used to confirm correct electrode positioning. The tip of the Levin-Cosman cordotomy electrode was stimulated at approximately $0.1 \mathrm{~V}$, which did not result in a physiological change, probably because of the deep sedation. A single lesion was created at $90^{\circ} \mathrm{C}$ for 90 seconds. The patient was awakened from general anesthesia and extubated without problem. Immediately after the procedure, she experienced relief from right hemicranial pain; the relief continued through her 3-month follow-up visit.

\section{Discussion}

Trigeminal tractotomy first dates to 1938, when Sjöqvist ${ }^{8}$ performed a transverse stab incision of the trigeminal tract to relieve facial pain. Schvarcz ${ }^{6,7}$ then transformed the procedure into a percutaneous approach by using a special stereotactic apparatus designed for craniocervical junction lesioning such as tractotomy and myelotomy. Lesioning of the tract and nucleus, which is universally ablated because of its proximity to the tract, is essential for treating cancerassociated pain of the face and neuropathic facial pain syndromes or anesthesia dolorosa. A more radical version of lesioning of the tract and nucleus is the caudalis root entry zone operation; tractotomy-nucleotomy is therefore considered a miniature caudalis dorsal root entry zone ablation.

In 1989, Kanpolat et al. ${ }^{2}$ first introduced the idea of CT-guided percutaneous ablation of the upper spinal cord for pain, including CT-guided trigeminal tractotomy, which now constitutes the most commonly used tractotomy-nucleotomy method. Percutaneous tractotomy is performed under local anesthesia, and needle position is verified by the awake patient after stimulation-induced paresthesia of the face. The introduction of intraoperative imaging, which is safe for patients under general anes- thesia, enabled percutaneous CT-guided procedures to be performed in anesthetized patients. ${ }^{1}$ In the cases described here, performing the procedure under local anesthesia was not possible because of the extreme facial allodynia and hyperalgesia that the patients experienced in the prone position. Lateral positioning is an option; however, we prefer direct prone positioning with the head in slight flexion to ease anatomical guidance of the needle entry site and trajectory of the needle tip into the spinal cord. Conscious sedation or monitored anesthesia were not considered because the patients might move during the procedure, and any head movement while the tip of the electrode is in the upper spinal cord could result in complications such as hemiparesis or hemiplegia. Because, in our opinion, a full open caudalis dorsal root entry zone procedure was not indicated in Case 1, for added safety we therefore elected to perform percutaneous intraoperative CT-guided tractotomy-nucleotomy with intraoperative neuromonitoring. We argue that in patients for whom the anatomical target is very well characterized, such as the trigeminal tract, a lesion could be made without verification from the awake patient, provided that real-time intraoperative verification of the electrode position is possible.

In the patients reported on here, a CereTom CT scanner, which is specifically designed for cranial imaging and not spinal imaging, was used. The physique of the patient in Case 1 (a long and thin neck) was such that use of the CereTom CT scanner in 3-point fixation was favorable. The physique of the patient in Case 2 was less favorable, necessitating the use of a scan board designed to fit in the CereTom CT scanner. If these scanners are available, we recommend use of a whole-body or spine scanner such as the O-arm (Medtronic) or BodyTom (NeuroLogica Corp.) for CT-guided tractotomy-nucleotomy procedures.

After surgery, the patient in Case 1 experienced temporary ataxia of the left arm and leg and numbness of the left arm, which significantly improved after 2 days, and he reported complete relief of his postherpetic neuralgia pain. At the most recent visit, 6 months after surgery, the patient reported significant pain relief. The patient in Case 2 did not experience any adverse effects from the lesioning and was completely pain free 3 months after surgery.

The largest series of CT-guided tractotomy-nucleotomy procedures has been reported by Kanpolat et al. ${ }^{3}$ and Raslan. ${ }^{5}$ Use of general anesthesia for this procedure has not been reported. To our knowledge, this is the first report of percutaneous intraoperative CT-guided tractotomy-nucleotomy in fully anesthetized patients. Given the preliminary safety and success for this procedure in our 2 patients, we advocate the use of general anesthesia for CT-guided tractotomy-nucleotomy procedures.

\section{Conclusions}

Use of intraoperative CT imaging to gain adequate radiologic verification of electrode position enables percutaneous CT-guided tractotomy-nucleotomy to be performed on anesthetized patients. Percutaneous intraoperative CT-guided tractotomy-nucleotomy is an effective technique for relief of postherpetic neuralgia and postoperative hemicranial neuralgia. 


\section{Disclosure}

The authors report no conflict of interest concerning the materials or methods used in this study or the findings specified in this paper.

Author contributions to the study and manuscript preparation include the following. Conception and design: Raslan. Acquisition of data: Raslan, Thompson. Analysis and interpretation of data: all authors. Drafting the article: all authors. Critically revising the article: all authors. Reviewed submitted version of manuscript: all authors. Approved the final version of the manuscript on behalf of all authors: Raslan.

\section{Acknowledgments}

The authors thank Shirley McCartney, Ph.D., for editorial assistance and Andy Rekito, M.S., for figure preparation.

\section{References}

1. Collins KL, Patil PG: Flat-panel fluoroscopy O-arm-guided percutaneous radiofrequency cordotomy: a new technique for the treatment of unilateral cancer pain. Neurosurgery $72(1$ Suppl Operative):27-34, 2013

2. Kanpolat Y, Deda H, Akyar S, Cağlar S, Bilgiç S: CT-guided trigeminal tractotomy. Acta Neurochir (Wien) 100:112-114, 1989

3. Kanpolat Y, Kahilogullari G, Ugur HC, Elhan AH: Comput- ed tomography-guided percutaneous trigeminal tractotomynucleotomy. Neurosurgery 63 (1 Suppl 1):ONS147-ONS155, 2008

4. Levin-Cosman AB, Cosman ER: Thermocouple-monitored cordotomy electrode. Technical note. J Neurosurg 53:266268, 1980

5. Raslan AM: Percutaneous computed tomography-guided radiofrequency ablation of upper spinal cord pain pathways for cancer-related pain. Neurosurgery 62 (3 Suppl 1):226-234, 2008

6. Schvarcz JR: Postherpetic craniofacial dysaesthesiae: their management by stereotaxic trigeminal nucleotomy. Acta Neurochir (Wien) 38:65-72, 1977

7. Schvarcz JR: Stereotactic trigeminal tractotomy. Confin Neurol 37:73-77, 1975

8. Sjöqvist O: Studies on pain conduction in the trigeminal nerve: a contribution to the surgical treatment of facial pain. Acta Psychiatr Neurol Scand Suppl 17:1-139, 1938

Manuscript submitted May 14, 2013.

Accepted June 12, 2013.

Please include this information when citing this paper: DOI: 10.3171/2013.6.FOCUS13218.

Address correspondence to: Ahmed M. Raslan, M.D., Department of Neurological Surgery, Oregon Health \& Science University, Mail Code CH8N, 3303 SW Bond Ave., Portland, OR 97239-3098. email: raslana@ohsu.edu. 